\title{
ACOUSTOELECTRONIC SENSORS
}

\section{АКУСТОЕЛЕКТРОННI СЕНСОРИ}

PACS: 42.70.Ce; 42.55.Lt; 77.70.+a, 85.50.-n

\section{МНОГОФУНКЦИОНАЛЬНЫЙ КВАРЦЕВЫЙ СЕНСОР}

\section{В. Ф. Косоротов, Л. В. Щедрина, С. К. Скляренко}

Институт физики НАН Украины

46, пр. Науки, 03028, Киев, Украина

+38(044)5257942; +38(044)5251589

1shched@iop.kiev.ua, kosorot@iop.kiev.ua

\section{МНОГОФУНКЦИОНАЛЬНЫЙ КВАРЦЕВЫЙ СЕНСОР}

\author{
В. Ф. Косоротов, Л. В. Щедрина, С. К. Скляренко
}

\begin{abstract}
Аннотация. Представленное многофункциональное пироэлектрическое устройство - ваттджоульметр объединяет в себе функции измерителя мощности и измерителя энергии излучения. Разработанная технология создания пироэлектрических сенсоров, основанная на учете пространственного распределения электрического потенциала в неполярных пьезоэлектрических кристаллах в условиях неоднородного нагрева, обеспечивает необходимые требования для формирования двух независимых измерительных каналов на одном чувствительном элементе. Одновременное измерение мощности и энергии импульса излучения не требует дополнительной электронной обработки выходного сигнала и осуществляется без погрешностей измерения, связанных с наличием температуры перехода в сегнетоэлектриках.

Ключевые слова: третичный пироэлектрический эффект, пространственно неоднородный нагрев, индуцированная пироактивность, кварц, пироэлектрический сенсор
\end{abstract}

\section{БАГАТОФУНКЦІОНАЛЬНИЙ КВАРЦОВИЙ СЕНСОР}

\section{В. П. Косоротов, Л. В. Щедріна, , С. К. Скляренко}

Анотація. Представлений багатофункціональний піроелектричний пристрій - ватджоульметр поєднує в собі функції вимірювача потужності й вимірювача енергії випромінювання. Розроблена технологія створення піроелектричниих сенсорів, що заснована на урахуванні

(C) В. П. Косоротов, Л. В. Щедріна, С. К. Скляренко, 2016 
просторового розподілу електричного потенціалу в неполярних п'єзоелектричних кристалах в умовах неоднорідного нагрівання, забезпечує необхідні вимоги для формування двох незалежних вимірювальних каналів на одному чутливому елементі. Одночасний вимір потужності й енергії імпульсу випромінювання не потребує додаткової електронної обробки вихідного сигналу й здійснюється без похибок, пов'язаних з наявністю температури переходу в сегнетоелектриках.

Ключові слова: третинний піроелектричний ефект, просторово неоднорідне нагрівання, індукована піроактивність, кварц, піроелектричний сенсор

\title{
MULTIFUNCTIONAL QUARTZ SENSOR
}

\section{F. Kosorotov, L. V. Shchedrina, S. K. Sklyarenko}

\begin{abstract}
Elaborated multifunctional pyroelectric device - watt/joule meter combines the functions of radiation power meter and radiation energy meter. Proposed techniques of pyroelectric sensors based on a consideration of the spatial electric potential distribution in nonpolar piezoelectric crystals under inhomogeneous heating conditions provides needed requirements for a formation of two independent measuring channels on common sensitive element. Simultaneous measuring pulse power and energy does not require additional electronic treatment of output signal and is carried out without measurement errors associated with the presence of transition temperature in ferroelectrics.
\end{abstract}

Keywords: tertiary pyroelectric effect, spatially nonuniform heating, induced pyroelectric response, quartz, pyroelectric sensor

\section{1. Введение}

Современное состояние ИК оптоэлектроники в значительной степени определяется качеством используемых материалов, среди которых важнейшими являются пироэлектрические материалы. Получение новых материалов, обладающих уникальными и управляемыми свойствами, требует значительных капиталовложений. Альтернативное решение этой проблемы заключается в поиске новых, перспективных для дальнейшего развития пироэлектрического приборостроения, свойств в традиционных материалах.

“Классические" пироэлектрические устройства, работающие на первичном и вторичном пироэлектрическом эффектах, перестают быть эффективными при измерении мощного излучения лазеров из-за перегрева чувствительного элемента в процессе измерения и наличия в нем температуры перехода. В связи с этим нами разрабатываются пироэлектрические сенсоры (ПС), базирующиеся на использовании индуцированных пироактивных сред. Неполярные пьезоэлектрические кристаллы, не обладающие пироэлектрическими свойствами, в усло- виях пространственно неоднородного нагрева приобретают пироактивность за счет третичного пироэлектрического эффекта (ТПЭ) [1].

В настоящей работе представлен функциональный ряд новых сенсорных устройств на основе индуцированных кварцевых пироактивных сред, который позволяет решать не только задачи, связанные с мониторингом интенсивных потоков излучения, но и конструировать многофункциональные устройства на основе ТПЭ. Отличительной особенностью этих сенсоров является то, что они реализованы на основе $X_{3}$-среза кварца, который ранее считался непригодным для метрологии лазерного излучения, поскольку все его пьезомодули $d_{3 \lambda}$ равны нулю. Другой их отличительной особенностью является то, что они используются в условиях почти полной прозрачности кристалла, что значительно повышает пределы измеряемых энергетических характеристик излучения.

В кварце (класс $D_{3}$ ), априори не обладающем спонтанным электрическим моментом и имеющем высокую радиационную стойкость и прозрачность в видимой и ближней ИК об- 
ластях спектра, отсутствуют нелинейные искажения выходного сигнала, характерные для полярных кристаллов в силу наличия в последних температуры перехода. Пироактивные свойства в кварце индуцируются в процессе облучения и связаны с его пространственно неоднородным нагревом. Принципиальным является то, что для проявления ТПЭ, являющегося откликом пьезоэлектрической поляризации кристалла в термодинамически неравновесных условиях, не требуется наличие особенного полярного направления в кристалле и поэтому все ацентричные кристаллы активны относительно проявления ТПЭ в них. При этом ТПЭ существует только в определенных срезах кварца и при определенной конфигурации температурного поля в нем.

Сложный характер пространственного распределения индуцированной поляризации определяется как способом нагрева кристалла, так и его кристаллографическим срезом. Поэтому главной задачей при создании ТПЭ сенсоров является установление связи конфигурации электрического поля в объеме термодинамически неравновесного кристалла со специально разработанной системой электродов для регистрации излучения.

\section{2. Особенности проявления индуциро- ванной пироактивности}

Все пироэлектрики в силу их слабой прозрачности в средней ИК-области спектра малопригодны для использования их в качестве чувствительных элементов сенсоров интенсивных потоков излучения. Поэтому первоначально сама идея использования материалов, не принадлежащих пироэлектрическим классам, в качестве пироактивных структур была предложена в нашей работе [2] с целью повышения предела измеряемых энергетических характеристик интенсивного лазерного излучения. Очевидно, что это становится возможным только на основе физического эффекта, проявляющегося в кристаллах, обладающих высоким уровнем прозрачности и высокой радиационной стойкостью в различных спектральных диапазонах. Был разработан сенсор на ориентированном монокристалле селенида цинка, относящемся к кристаллографическому классу $T_{d}$, [3], оказавшийся эффективным при измерении интенсивного излучения $\mathrm{CO}$ и $\mathrm{CO}_{2}$-лазеров с длиной волны, лежащей в области прозрачности этого кристалла. Достаточно слабое оптическое поглощение $\mathrm{ZnSe}$ (порядка $10^{-3} \mathrm{~cm}^{-1}$ ) на длине волны регистрируемого излучения обеспечило значительное увеличение верхней границы динамического диапазона: предельные проходные средняя и импульсная мощности оказались равными 3 кВт и 1 МВт, соответственно.

Помимо сенсоров проходного типа анализ пироактивности, индуцированной в ацентричном кристалле векторным тепловым воздействием, выявил возможности конструирования многофункциональных устройств на основе ТПЭ, позволяющих в едином масштабе времени проводить измерения нескольких характеристик потоков излучения. Разработанный нами первый ватт-джоульметр на основе неполярного $X_{1}$-среза ниобата лития [4] как раз и был таким устройством. При использовании этого сенсора для одновременного измерения мощности и энергии импульса излучения на одном чувствительном элементе не требуется дополнительной электронной обработки выходного сигнала. Ватт-джоульметр, описанный в [5], был разработан на основе $X_{2}$-среза кварца. Важно отметить, что оба эти сенсора были реализованы в условиях поверхностного поглощения излучения при изменении формы чувствительного элемента, вызванного градиентом температуры вдоль его толщины.

Отметим, что сенсор на основе $\mathrm{ZnSe}$ также можно использовать в качестве многофункционального приемного устройства, выполняющего одновременно функции выходного оптического элемента и контрольно измерительного устройства. Если реализовать данный сенсор на оптическом элементе, являющемся выходным окном лазера, то это устройство представляет собою одновременно и приемник параметров выходного излучения.

\section{3. Пироэлектрический эффект в $X_{3}$-срезе кварца}

Как указывалось в [6], $X_{3}$-срез вызывает прежде всего теоретический интерес. Его изучение необходимо для подтверждения ряда важных положений, вытекающих из теории 
ТПЭ. Результаты изучения пироактивности в указанном срезе фактически переводят этот кристалл из чисто модельного в разряд одного из самых перспективных материалов для пироэлектрического приборостроения. Теоретические и экспериментальные исследования были проведены для одномерного температурного поля, характеризующегося цилиндрической симметрией. Экспериментальный образец из кристаллического кварца в виде его $X_{3}$ - среза имел форму круглой пластинки диаметром 20 мм и толщиной $\ell=0,5$ мм. Чувствительный элемент подвергался осесимметричному нагреву синусоидально модулированным с частотой $\omega$ пучком гелий-неонового лазера радиуса $r_{0}$. С целью обеспечения осесимметричного нагрева и высокой чувствительности метода образец чернился, а частота модуляции $\omega$ и толщина диска $\ell$ выбирались такими, чтобы длина температурной волны $\lambda_{T}=\sqrt{2 a / \omega}>\ell$. Это условие обеспечивало независимость температурного поля по толщине кристалла и одновременно почти полное поглощение падающего излучения. Реализация осесимметричного нагрева $\left(r_{\mathrm{o}}<<R\right.$, где $R$ - радиус диска) обуславливает наличие двух областей с различным распределением стационарной части температуры в них при радиальном температурном градиенте.

Анализ распределения прироста температуры в кристалле $\theta(r, \omega, t)$ показывает, что эта функция в области $r<r_{\text {o }}$ слабо зависит от радиуса $r$, а в области $r>r_{\text {о }}$ спадает до нуля по закону $e^{-\left(r-r_{\mathrm{o}}\right) / \lambda_{T}}$. Таким образом, в области $r>r_{\text {。 }}$ кристалл остается практически холодным в процессе его облучения. Отметим, что для разрушения полярного состояния полного выравнивания температуры по кристаллу не требуется, поскольку термоупругие напряжения, вызывающие ТПЭ, исчезают уже при линейной зависимости температуры от координат [7].

Теоретическое рассмотрение базируется на симметрийном анализе механического состояния пластинки в радиально неоднородных температурных полях, развитом в [8]. Для $X_{3}-$ среза кварца, плоскость которого перпендикулярна оси третьего порядка, получен ряд новых результатов. Так, оказалось, что если даже в группе симметрии внешнего воздействия $G$ и содержится ось третьего порядка, но для проявления полярного состояния вовсе не требуется наличия особенного полярного направления. И вектор индуцированной поляризации не совпадает с направлением оси 3.

Итак, в $X_{3}$ - срезе кварца продольный пьезоэлектрический эффект (следовательно, и продольный ТПЭ) строго запрещен во всем объеме. Существовать может только поперечный ТПЭ. В нагретой области пластинки $\left(r<r_{\mathrm{o}}\right)$ группа симметрии внешнего воздействия $G=32$. Это означает, что при тепловом воздействии на кристалл группа его симметрии в этой зоне не меняется и, следовательно, поляризация отсутствует.

В холодной области полярное состояние характеризуется группой симметрии внешнего воздействия $G \supseteq 3$. Казалось бы, вектор поляризации $\boldsymbol{P}$ должен быть направлен вдоль особенного полярного направления, содержащегося в группе $G \supseteq 3$, так как возмущенный кристалл с такой группой симметрии относится к пироэлектрическому классу. Однако продольный пироэффект, как отмечалось, по-прежнему запрещен, а имеет место поперечный эффект. То есть вектор индуцированной поляризации оказывается вообще перпендикулярен особенному полярному направлению. Результат неожиданный — вектор индуцированной поляризации может не совпадать с особенным полярным направлением, содержащемся в группе, описывающей симметрию кристалла в поле внешнего воздействия.

Полярное состояние реализуется в плоскости среза $X_{1} X_{2}$, где лежат как полярные, так и неполярные направления невозмущенного кристалла, а действующие термоупругие напряжения, вызванные радиальным температурным градиентом, индуцируют в ней поляризацию. И это полярное состояние является инвариантным относительно элементов симметрии группы $G \supseteq 3$. Вычисленное распределение радиальной компоненты поляризации $P_{r}(r, \varphi)$, пропорциональное $\sin 3 \varphi$, является инвариантным относительно всех преобразований группы $G \supseteq 3$. В следующем разделе будет показано, что эти результаты симметрийного анализа хорошо согласуются с экспериментальными данными, впервые получившими объяснение на его основе. 


\section{4. Сенсоры на основе пироактивного кварца}

Развитый симметрийный подход оказывается эффективным методом определения основных особенностей пространственного распределения поляризации, информация о которых является необходимой при разработке физических принципов построения нового класса ПС, действующих на основе индуцированной пироактивности в ацентричных кристаллах. Принципы организации устройств, основой которой является связь конфигурации электрического поля в объеме термодинамически неравновесного кристалла со специально разработанной системой электродов для регистрации излучения, позволили предложить и реализовать два класса сенсоров на основе $X_{3}$-среза кварца. Первый класс - сенсоры для мониторинга интенсивных потоков излучения в видимой и ближней ИК областях спектра, второй - ватт-джоульметр. Для их реализации необходимо знание распределения электрического потенциала на чувствительном элементе, которое в случае неоднородного нагрева кристалла связано с распределением термоупругих напряжений. В данном срезе кварца имеет место только поперечная компонента электрического поля, лежащая в плоскости пластинки в ее холодной области, обусловленная связанным зарядом на боковой поверхности диска с угловой зависимостью аналогичной распределению радиальной компоненты поляризации. Это дает возможность измерить радиальное распределение потенциала, связанного с этим зарядом, и реализовать сенсор интенсивных потоков излучения. Отметим, что впервые поперечный ТПЭ с аналогичной угловой зависимостью был обнаружен в кристалле селенида цинка в срезе, перпендикулярном кристаллографическому направлению [111] [3]. Схема регистрации выходного сигнала в кварце выглядит следующим образом. На боковую поверхность чувствительного элемента наносилась система из 12 изолированных друг от друга электродов, что позволило измерить угловое распределение потенциала с дискретностью в $30^{\circ}$. Все участки боковой поверхности пластинки, на которых вектор поляризации имеет одинаковое направление, соединяются в одну точку, а участки с противоположным знаком поляризации на них - в другую точку. Возникающая на электродах отличная от нуля разность потенциалов содержит информацию о падающем потоке излучения. Такая пара электродов, будучи подсоединенной к согласующему каскаду, обеспечивает регистрацию выходного сигнала пропорционально падающей мощности излучения. Экспериментальная структура пространственного распределения электрического потенциала, представленная на Рис. 1, хорошо коррелирует с вычисленным распределением радиальной компоненты поляризации $P_{r}(r, \varphi)$.

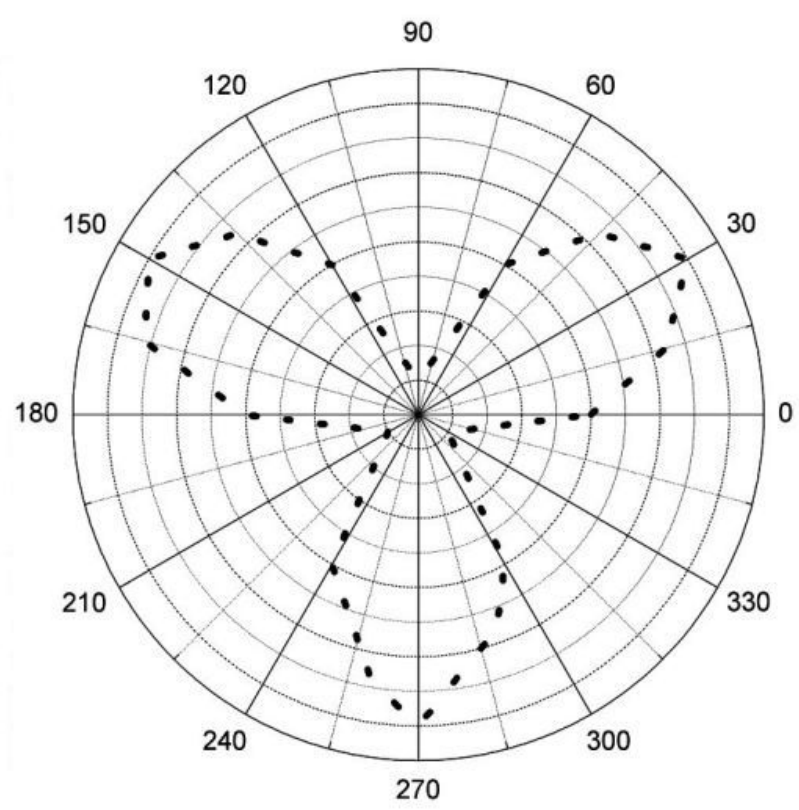

Рис. 1. Экспериментальная структура пространственного распределения электрического потенциала в $\mathrm{X}_{3}$-срезе кварца.

Опишем второй тип разработанного устройства на $X_{3}$-срезе кварца. С учетом полученных теоретических и экспериментальных результатов можно предложить следующую схему реализации многофункционального ПС для одновременного измерения энергетических характеристик излучения. Соединенные в один конец участки боковой поверхности пластинки, на которых вектор поляризации обладает одинаковым знаком, образуют один из пары измерительных электродов. В качестве второго электрода используется "земля" в центре диска. Такая пара электродов, под- 
соединенная к согласующему каскаду с малым входным сопротивлением, обеспечивает регистрацию выходного сигнала пропорционально падающей мощности излучения. Канал измерения энергии создается аналогичным способом при использовании участков с противоположным знаком поляризации на них и большим входным сопротивлением согласующего каскада. На Рис. 2 представлены осциллограммы выходных сигналов каналов измерения мощности (верхняя кривая) и энергии (нижняя кривая). Коэффициент преобразования для канала измерения мощности был равен $3 \times 10^{-3} \mathrm{~B} / \mathrm{BT}$, для канала энергии $2 \times 10^{-1} \mathrm{~B} /$ Дж в диапазоне частот от 0,2 до 5 Гц. Минимально измеряемые значения мощности и энергии, соответственно, были равны $10^{-2} \mathrm{BT}, 10^{-4}$ Дж.
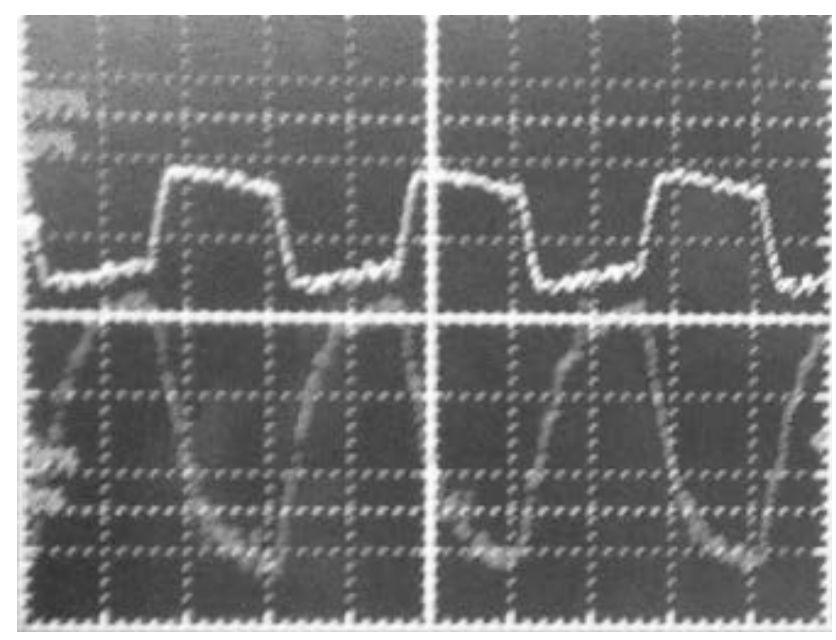

Рис. 2. Осциллограммы выходных сигналов каналов измерения мощности (верхняя кривая) и энергии (нижняя кривая).

Оба устройства могут быть идеальными измерителями соответствующих параметров излучения при независимости их коэффициентов преобразования от диаметра пучка излучения. Однако, как видно из Рис. 3, представляющего зависимости выходного сигнала от диаметра пучка $D$ для обоих каналов, этому условию удовлетворяет только канал измерения мощности.

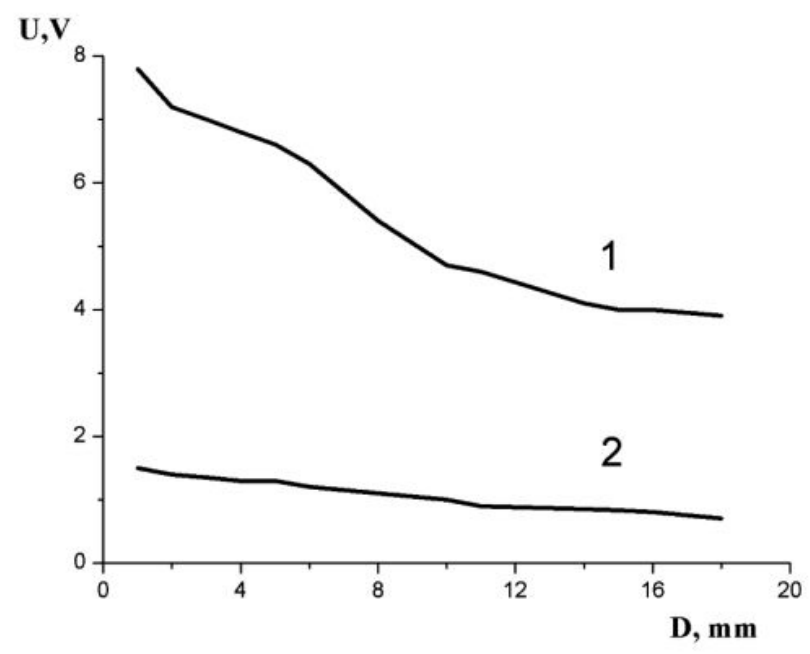

Рис. 3. Зависимости выходного сигнала от диаметра пучка излучения (D) для канала измерения энергии (кривая 1) и мощности (кривая 2).

Физический механизм, объясняющий эту особенность, состоит в следующем. Достоверное измерение энергии достигается только по истечении времени $t>\tau_{E}\left(\tau_{E}-\right.$ электрическая постоянная времени), за которое при малых частотах модуляции $\lambda_{T}>\ell$. Тогда при малых значениях диаметра пучка удовлетворяется условие $\lambda_{T}>r_{\text {o }}$, что приводит к сильному размытию засвечиваемой области и значительная часть энергии попадает на нерабочие для этого канала участки диска. В канале измерения мощности эти ограничения отсутствуют и его коэффициент преобразования почти не зависит от $D$.

\section{Список использованной литературы}

[1]. V. F. Kosorotov, L. S. Kremenchugskij, V. B. Samoilov, L. V. Shchedrina. Pyroelectric Effect and Its Practical Applications. Naukova Dumka, Kiev, 224 p. (1987).

[2]. V. F. Kosorotov, L. S. Kremenchugskij, L. V. Levash, L. V. Shchedrina. Tertiary pyroelectric effect in lithium niobate and lithium tantalate crystals // Ferroelectrics, 70, N 1/2, pp. 27 -37 (1986).

[3]. V. F. Kosorotov, L. V. Shchedrina, L. V. Levash, Yu. A. Zagoruiko et al. Power sensors, based on the tertiary pyroelectric effect, combined with exit windows of $\mathrm{CO}$ 
and $\mathrm{CO}_{2}$ lasers // Quantum Electronics, 21(6), pp. 543-545 (1994).

[4]. V. F. Kosorotov, L. V. Shchedrina, L. V. Levash. Multifunctional devices based on induced polar states // Semiconductor Physics, Quantum Electronics \& Optoelectronics, 8, № 3, pp. 60-65 (2005).

[5]. V. F. Kosorotov, L. V. Shchedrina. New functional capabilities of quartz for laser parameters measurements // Quantum Electronics, 40, № 3, pp. 271-275 (2010).

[6]. V. F. Kosorotov, L. V. Shchedrina, S. K. Sklyarenko. Quartz-based pyroelec- tric sensor // Sensor Electron. Microsys. Tech., 12, № 1, pp. 20-25 (2015).

[7]. Yu. P. Sirotin, M. P. Shaskolskaya. Fundamentals of Crystallophysics, Nauka, Moscow, 640 p. (1979).

[8]. V. F. Kosorotov, L. V. Shchedrina. Symmetric analysis of induced pyroactyvity in radially inhomogeneous temperature fields // Ukrainian Journal of Physics, 54, No. 1-2, $171-176$ (2009).

Стаття надійшла до редакції 25.05.2016 p.

PACS: 42.70.Ce; 42.55.Lt; 77.70.+a, 85.50.-n

\title{
MULTIFUNCTIONAL QUARTZ SENSOR
}

\author{
V. F. Kosorotov, L. V. Shchedrina, S. K. Sklyarenko \\ Institute of Physics, NAS of Ukraine \\ Prospect Nauky, 46, 03028 Kyiv, Ukraine
}

\begin{abstract}
Summary
The present work is devoted to experimental and theoretical investigation of the tertiary pyroelectric effect induced in quartz crystal by a spatially nonuniform heating. The pyroelectric response of quartz being nonpolar piezoelectric crystal, is induced during irradiation. Offering high radiation hardness, good transmission in the visible and near-JR spectral regions and temperature-stable parameters determining TPE sensitivity, quartz as an induced pyroelectric response material has been used under making novel pyroelectric sensors. The developed devices based on induced quartz pyroelectric response media allows one not only to solve problems related to high intensity laser output monitoring but also to design TPE-based multifunctional devices used to measure temporal and energetic characteristics of laser radiation simultaneously.
\end{abstract}

Keywords: tertiary pyroelectric effect, spatially nonuniform heating, induced pyroelectric response, quartz, pyroelectric sensor 
PACS: 42.70.Ce; 42.55.Lt; 77.70.+a, 85.50.-n

\title{
БАГАТОФУНКЦІОНАЛЬНИЙ КВАРЦОВИЙ СЕНСОР
}

\author{
В. П. Косоротов, Л. В. Щедріна, С. К. Скляренко \\ Інститут фізики НАН України \\ 46, пр. Науки, 03028, Київ, Україна \\ +38(044)5257942; +38(044)5251589 \\ 1shched@iop.kiev.ua,kosorot@iop.kiev.ua
}

\begin{abstract}
Реферат
Робота присвячена теоретичному та експериментальному дослідженню третичного піроелектричного ефекту, що розвивається в умовах просторово неоднорідного нагріву в кристалі кварцу. Піроактивні властивості кварцу, що є неполярним п'єзоелектричним кристалом, індукуються в процесі його опромінення. Кварц, завдяки високій радіаційній стійкості та прозорості у видимій та ближній ІЧ областях спектру, температурно стабільним параметрам, що визначає ТПЕ чутливість, був використаний в якості індукованого піроактивного матеріалу при створенні піроелектричних сенсорів нового класу. Розроблені пристрої на основі піроактивних кварцових середовищ дозволяють вирішувати не тільки завдання, пов'язані з моніторингом інтенсивних потоків випромінювання, але і конструювати багатофункціональні сенсори для одночасного вимірювання тимчасових і енергетичних характеристик випромінювання.

Ключові слова: третинний піроелектричний ефект, просторово неоднорідне нагрівання, індукована піроактивність, кварц, піроелектричний сенсор
\end{abstract}

